\title{
Pacific
}

Journal of

Mathematics

\section{THE COVERS OF A NOETHERIAN MODULE}

\author{
JIAN-JUN CHUAI
}




\title{
THE COVERS OF A NOETHERIAN MODULE
}

\author{
JIAN-JUN CHUAI
}

\section{In this paper we define the covers of a module and describe some of their applications.}

\section{Introduction.}

Let $R$ be a commutative ring and $A$ an $R$-module. A cover of $A$ is defined to be a subset $T$ of $\operatorname{Max}(R)$ satisfying that for any $x \in A, x \neq 0$, there is $M \in T$ such that $0:_{R} x \subseteq M$. If we denote by $J$ the intersection of all the maximal ideals belonging to $T$ and suppose that $A \neq 0$ is finitely generated, then we have $J A \neq A$. This generalises the Nakayama's lemma; if, in addition, $R$ is Noetherian, then $\bigcap_{n=1}^{\infty} J^{n} A=0$. This is a generalization of a well-known result. A key observation for the covers is that, in the case that $R$ is Noetherian and $A$ is finitely generated, there is a cover $T$ of $A$ which is itself a finite set. From this we have the following result: Let $R$ be a Noetherian ring. Then there is a finite number of maximal ideals $M_{1}, \ldots, M_{m}$ of $R$ such that $\bigcap_{n=1}^{\infty} J^{n}=0$, where $J=\bigcap_{i=1}^{m} M_{i}$. This generalises the Krull's theorem for Jacobson radicals. Using this result we can embed the Noetherian ring $R$ in the $J$-adic completion $\widehat{R}$ of $R$, which is a complete semi-local Noetherian ring; besides, if $R$ is a Cohen-Macaulay (C-M for short) ring, then $\widehat{R}$ is a $\mathrm{C}-\mathrm{M}$ ring. We also use the covers to deal with the maximal component of a finitely generated module over a Noetherian ring, which was introduced by Matlis in [3].

Throughout the paper, $R$ will denote a (non-trivial) commutative ring with identity. Also, if $T$ is a subset of $\operatorname{Max}(R)$ we denote by $\cap T$ (resp. $\cup T$ ) the intersection (resp. union) of all the maximal ideals belonging to $T$.

\section{The covers.}

In this section we define the covers of a module and generalise some known results.

Definition. Let $A$ be an $R$-module. A subset $T$ of $\operatorname{Max}(R)$ is called a cover of $A$ if for any $x \in A, x \neq 0$, there is $M \in T$ such that $0:_{R} x \subseteq M$.

Clearly, if $T$ is a cover of $A$ and $B$ is a submodule of $A$, then $T$ is a cover of $B$. If $T$ is a cover of $A$ and $T \subseteq T^{\prime} \subseteq \operatorname{Max}(R)$, then $T^{\prime}$ is a cover of $A$. We 
say that $T$ is a finite cover of $A$, or $A$ has a finite cover $T$, if $T$ is a cover of $A$ and $T$ is itself a finite set. If $T$ is a cover of $A$, we also say that $T$ covers $A$.

Lemma 2.1. Let $T$ be a cover of $A$. Then each $r \in R-\cup T$ is A-regular. Indeed if $a \in A-\{0\}$ and $r a=0$, then $r \in\left(0:_{R} a\right) \subseteq M$ for some $M \in T$, a contradiction.

Proposition 2.2. Let $A \neq 0$ be a finitely generated $R$-module and $T$ a cover of $A$. Then $J A \neq A$, where $J=\cap T$.

Proof. Suppose that $J A=A$, then there is $r \in J$ such that $(1+r) A=0$, which contadicts Lemma 2.1.

Proposition 2.3. Let $A$ be an $R$-module, $T$ a cover of $A$, and $I \nsubseteq 0:_{R} A$ an ideal of $R$. Set $J=\cap T$. If $A / 0:_{A} I$ is finitely generated, then $J A+\left(0:_{A}\right.$ $I) \neq A$.

Proof. Since $I \nsubseteq 0:_{R} A, A / 0:_{A} \neq 0$. Let $\bar{x} \in A / 0:_{A} I$ and $\bar{x} \neq 0$. Then $0:_{R} \bar{x}=\left(0:_{A} I\right):_{R} x \subseteq 0:_{R} I x$. Since $x \notin 0:_{A} I, I x \neq 0$. Take $r \in I$ such that $r x \neq 0$, then $0:_{R} \bar{x} \subseteq 0:_{R} r x$. it follows that $T$ is a cover of $A / 0:_{A} I$. By Proposition 2.2, $J\left(A / 0:_{A} I\right) \neq A / 0:_{A} I$, hence $J A+\left(0:_{A} I\right) \neq A$.

Proposition 2.4. Let $R$ be a Noetherian ring, $A$ a finitely generated $R$ module, $T$ a cover of $A$, and $I \subseteq \cap T$ an ideal of $R$. Then $\bigcap_{n=1}^{\infty} I^{n} A=0$.

Proof. Set $\bigcap_{n=1}^{\infty} I^{n} A=B$. By Krull's theorem, there is $r \in I$ such that $(1+$ $r) B=0$. From Lemma $2.1, B=0$.

Proposition 2.5. Let $T$ be a finite subset of $\operatorname{Max}(R)$ and $A$ an $R$-module. Set $J=\cap T$. If $\bigcap_{n=1}^{\infty} J^{n} A=0$, then $T$ is a cover of $A$.

Proof. If it were not true, there would be a non- zero element $x$ of $A$ such that for any $M \in T, 0:_{R} x \nsubseteq M$. Thus for any integer $n>0$ we have $\left(0:_{R} x\right)+M^{n}=R$, so $M^{n} x=R x$. It then follows that $J^{n} x=R x$, and thus $\bigcap_{n=1}^{\infty} J^{n} A \neq 0$, a contradiction.

Let $R$ be a Noetherian ring and $A$ a finitely generated $R$-module. We know that $\operatorname{Ass}(A)$ is a finite set. Let $\operatorname{Ass}(A)=\left\{P_{1}, \ldots, P_{n}\right\}$. Choose a finite subset $T$ of $\operatorname{Max}(R)$ in such a way that for any $P_{i}$, there is $M_{i} \in T$ such that $P_{i} \subseteq M_{i}$. Since for any $x \in A, x \neq 0$, there is $P_{i}$ such that $0:_{R} x \subseteq P_{i}$, it follows that $T$ is a finite cover of $A$. Hence finite covers exist for any finitely 
generated module over a Noetherian ring. In particular, any Noetherian ring (as a module over itself) has finite covers.

As a consequence of the above remarks and Proposition 2.4 we have the following theorem.

Theorem 2.6. Let $R$ be a Noetherian ring and $A$ a finitely generated $R$ module. Then there is a finite subset $T$ of $\operatorname{Max}(R)$ such that $\bigcap_{n=1}^{\infty} J^{n} A=0$, where $J=\cap T$. In particular, if $A=R, \bigcap_{n=1}^{\infty} J^{n}=0$.

It is clear that if $R$ is a Noetherian ring and $A$ is a finitely generated $R$-module, then for any cover $T$ of $A$ we have $T \supseteq \operatorname{Ass}(A) \cap \operatorname{Max}(R)$.

In general, if $T$ is a cover of the module $A$ and $B$ is a submodule of $A, T$ is not a cover of $A / B$. For example, if $T$ is a cover of the ring $R$ and $T \neq \operatorname{Max}(R)$, then for any $M \in \operatorname{Max}(R)-T, T$ is not a cover of $R / M$.

Proposition 2.7. Let $R$ be a Noetherian ring, $A$ a finitely generated $R$ module, $B$ a submodule of $A$, and $T$ a finite cover of $A$. Then $T$ is a cover of $A / B$ if and only if $B$ is a closed submodule of $A$ in the $J$-adic topology, where $J=\cap T$.

Proof. Suppose first that $B$ is closed, then we have $\bigcap_{n=1}^{\infty}\left(J^{n} A+B\right)=B$, so $\bigcap_{n=1}^{\infty} J^{n}(A / B)=0$. By Proposition $2.5, T$ is a cover of $A / B$. Conversely, if $T$ is a cover of $A / B$, then $\bigcap_{n=1}^{\infty} J^{n}(A / B)=0$, by Proposition 2.4. So $\bigcap_{n=1}^{\infty}\left(J^{n} A+B\right)=B$, and hence $B$ is closed.

Proposition 2.8. Let $R$ be a Noetherian ring, $A$ a finitely generated $R$ module, $B$ a submodule of $A$, and $I$ an ideal of $R$. Then it is possible to choose a finite subset $T$ of $\operatorname{Max}(R)$ such that $\bigcap_{n=1}^{\infty}\left(J^{n} A+I^{s} B\right)=I^{s} B$, for all $s \geq 0$, where $J=\cap T$.

Proof. By [5, Theorem 5.5(1)], the sequence $\operatorname{Ass}\left(A / I^{s} B\right)$ is constant for large $s$, thus the set $\bigcup_{s=0}^{\infty} \operatorname{Ass}\left(A / I^{s} B\right)$ is finite. Hence it is possible to choose a finite subset $T$ of $\operatorname{Max}(R)$ in such a way that $T$ covers all $A / I^{s} B$. By Proposition 2.4, the Proposition follows.

\section{The maximal component of a Noetherian module.}

Throughout this section and the next section the $\operatorname{ring} R$ will be Noetherian and the modules will be finitely generated. 
Let $A$ be an $R$-module and define $X(A)=\{x \in A \mid$ every prime ideal containing $0:_{R} x$ is maximal $\}$. Then $X(A)$ is a submodule of $A$. Matlis [3] called $X(A)$ the maximal component of $A$. By [3, Corollary $(3)$ ], $X(A)$ is the sum of all Artinian submodules of $A$, and hence is the largest Artinian submodule of $A$, since $A$ is Noetherian. Further, $X(A / X(A))=0$.

Chatters [4] gave a similar discussion for Noetherian rings (not necessary to be commutative).

From [3, Corollary (1)] and the fact that $X(A)$ has finite length we have the following result.

Theorem 3.1. Let $T$ be a finite cover of $A$. Set $J=\cap T$. Then $X(A)=$ $\bigcup_{n=1}^{\infty}\left(0:{ }_{A} J^{n}\right)$.

The following result is standard.

Lemma 3.2. Let $I$ be an ideal of $R$ and $A \neq 0$ an $R$-module. Then $\operatorname{dep}_{I}(A)>0$ if and only if $0:_{A} I=0$.

Theorem 3.3. Let $A$ be an $R$-module, not Artinian. Let $T$ be a finite cover of $A$ and set $J=\cap T$. Then $X(A)$ is the least element of the set

$$
S=\left\{B \mid B \quad \text { is a proper submodule of } A \text { and } \operatorname{dep}_{J}(A / B)>0\right\} .
$$

Proof. Since $A$ is not Artinian, $X(A)$ is a proper submodule of $A$. By Theorem 3.1 , we may assume that $X(A)=0:_{A} J^{N}$. Now

$$
0::_{A / X(A)} J=\left(X(A):_{A} J\right) / X(A)=0:_{A} J^{N+1} / 0:_{A} J^{N}=0 .
$$

From Lemma $3.2, \operatorname{dep}_{J}(A / X(A))>0$. Hence we have $X(A) \in S$. If $B$ is a proper submodule of $A$ satisfying that $\operatorname{dep}_{J}(A / B)>0$, again by Lemma $3.2,0:_{A / B} J=\left(B:_{A} J\right) / B=0$, i.e., $B:_{A} J=B$. Hence for any integer $n>0, B:_{A} J^{n}=B$. Thus we get that $B=B:_{A} J^{N} \supseteq 0:_{A} J^{N}=X(A)$, i.e., $X(A)$ is the least element of $S$.

Corollary 3.4. Let $A$ be a non-zero $R$-module and $T$ a finite cover of $A$. Set $J=\cap T$. Then $\operatorname{dep}_{J}(A)>0$ if and only if $X(A)=0$.

Let $T=\left\{M_{1}, \ldots, M_{n}\right\}$ be a finite cover of the $R$-module $A$. We want to find the relations between $X(A)$ and $X\left(A_{M_{i}}\right), 1 \leq i \leq n$. For any $P \in$ $\operatorname{Spec}(R)$, if $K$ is an $R_{P}$-submodule of $A_{P}$, denote by $K^{c}$ the contradiction of $K$ to $A$. We have $\left(K^{c}\right)_{P}=K$. If $B$ is a submodule of $A$, then $\left(B_{P}\right)^{c}=$ $\bigcup_{r \in R-P}\left(B:_{A} r\right)$. It is also easily checked that if $B$ is a submodule of $A$ and 
$K$ is an $R_{P}$-submodule of $B_{P}$, then $\left(K^{c} \cap B\right)_{P}=K$. It follows that if $B$ is an Artinian submodule of $A$, then $B_{P}$ is an Artinian submodule of $A_{P}$. In particular, we have $X(A)_{P} \subseteq X\left(A_{P}\right)$.

Theorem 3.5. Let $A$ be an $R$-module and $T=\left\{M_{1}, \ldots, M_{n}\right\}$ be a finite cover of $A$. Set $J=\cap T$. Then

$$
X(A)=\bigcap_{\imath=1}^{n} X\left(A_{M_{\imath}}\right)^{c} .
$$

Proof. Since $X(A) \subseteq\left(X(A)_{M_{i}}\right)^{c} \subseteq X\left(A_{M_{2}}\right)^{c}$ for all $i$, we have $X(A) \subseteq$ $\bigcap_{i=1}^{n} X\left(A_{M_{2}}\right)^{c}$. On the other hand, from Theorem 3.1 we can take a fixed integer $s>0$ such that $X\left(A_{M_{\imath}}\right)=0:_{A_{M_{2}}} M_{i}^{s} R_{M_{\imath}}$ for all $i$. Hence

$$
X\left(A_{M_{2}}\right)^{c}=\left(0:_{A_{M_{2}}} M_{i}^{s} R_{M_{i}}\right)^{c}=\left(\left(0:_{A} M_{i}^{s}\right)_{M_{2}}\right)^{c}=\underset{r \in R-M_{i}}{\cup}\left(\left(0:_{A} M_{i}^{s}\right):_{A} r\right) \text {. }
$$

If $x \in \bigcap_{i=1}^{n} X\left(A_{M_{i}}\right)^{c}$, then for each $i$ there is $r_{i} \in R-M_{i}$ such that $r_{i} M_{i}^{s} x=0$. Since $r_{\imath} R+M_{i}=R$, we have $M_{i}^{s+1} x=M_{i}^{s} x$. Thus

$$
M_{1}^{s+1} M_{2}^{s+1} x=M_{1}^{s+1} M_{2}^{s} x=M_{2}^{s} M_{1}^{s+1} x=M_{2}^{s} M_{1}^{s} x=M_{1}^{s} M_{2}^{s} x .
$$

Similarly we have $M_{1}^{s+1} \cdots M_{n}^{s+1} x=M_{1}^{s} \cdots M_{n}^{s} x$. So $J^{s+1} x=J^{s} x$, and hence $J^{s} x=0$ by Proposition 2.2. Thus $x \in 0:{ }_{A} J^{s} \subseteq X(A)$, and the proof is complete.

In the remainder of this section we consider modules over local rings.

Lemma 3.6. Let $(R, M)$ be a local ring ( $M$ is the unique maximal ideal of $R$ ) and $A$ an $R$-module. If $A$ is not Artinian, then $\operatorname{dim}(A)=\operatorname{dim}(A / X(A))$.

Proof. By the definitions of $\operatorname{dim}(A)$ and $\operatorname{dim}(A / X(A))$ we need to show that $\operatorname{rad}\left(0:_{R} A\right)=\operatorname{rad}\left(0:_{R}(A / X(A))\right)$. Clearly, we need only to show that $0:_{R}(A / X(A)) \subseteq \operatorname{rad}\left(0:_{R} A\right)$. This follows from the fact that if $r \in R$ such that $r A \subseteq X(A)$, then $r M^{s} A \subseteq M^{s} X(A)=0$ for some integer $s>0$, hence $r^{s+1} \in 0:_{R} A$.

Lemma 3.7. [6, p. 105]. Let $R$ be a local ring and $A$ an $R$-module. If $r_{1}, \ldots, r_{n}$ is an A-sequence, then

$$
\operatorname{dim}\left(A /\left(r_{1}, \ldots, r_{n}\right) A\right)=\operatorname{dim}(A)-n .
$$

Theorem 3.8. Let $(R, M)$ be a local ring and $A \neq 0$ an $R$-module. Then there is a strictly ascending chain $A_{1} \subset \cdots \subset A_{s}$ of submodules of $A$ such that

$$
\sum_{i=1}^{s} \operatorname{dep}\left(A / A_{\imath}\right)=\operatorname{dim}(A) .
$$


Proof. We use induction on $d=\operatorname{dim}(A)$. If $d=0$, then $R /\left(0:_{R} A\right)$ is Artinian. It follows that $0:_{R} A$ is $M$-primary, and hence $M^{r} \subseteq 0:_{R} A$ for some integer $r>0$. It is clear that $\operatorname{dep}(A)=0$, and we can take $s=1$ and $A_{1}=0$ in this case. If $d>0$, then $0:_{R} A$ is not $M$-primary, and thus $M^{n} \nsubseteq$ $0:_{R} A$ for any integer $n>0$. It then follows that $A \neq X(A)$, by Theorem 3.1. Since $X(A / X(A))=0, \operatorname{dep}(A / X(A))>0$, by Corollary 3.4. Take a maximal $A / X(A)$-sequence $x_{1}, \ldots, x_{n}$ and set $B=\left(x_{1}, \ldots, x_{n}\right) A+X(A)$. Further, set $A^{\prime}=A / X(A)$. From Lemma 3.7 and Lemma 3.6, $\operatorname{dim}(A / B)=$ $\operatorname{dim}\left(A^{\prime} /\left(x_{1}, \ldots, x_{n}\right) A^{\prime}\right)=\operatorname{dim}\left(A^{\prime}\right)-n=\operatorname{dim}(A)-n<\operatorname{dim}(A)$. By induction there is a strictly ascending chain $A_{2} / B \subset \cdots \subset A_{s} / B$ of submodules of $A / B$ such that $\sum_{i=2}^{s} \operatorname{dep}\left(A / A_{i}\right)=\operatorname{dim}(A / B)$. Set $A_{1}=X(A)$, then the submodules $A_{1}, \ldots, A_{s}$ satisfy the required conditions.

\section{The completions and embeddings.}

Proposition 4.1. Let $T$ be a finite cover of the Noetherian ring $R, I$ an ideal of $R$. If we consider $R$ with the I-adic topology, the following conditions are equivalent:

(1) $I \subseteq \cap T$;

(2) the zero ideal and every prime ideal contained in $\cup T$ is closed;

(3) $f^{-1}(M \widehat{R})=M$ for all $M \in T$, where $\widehat{R}$ is the I-adic completion of $R$ and $f: R \rightarrow \widehat{R}$ is the natural map.

Proof. (1) $\Rightarrow(2)$. Since $\bigcap_{m=1}^{\infty} I^{m}=0$ the zero ideal is closed. If $P \subseteq \cup T$ is a prime ideal, then $P \subseteq M$ for some $M \in T$. Since $\operatorname{Ass}_{R}(R / P)=\{P\}$, we see that $T$ is a cover of $R / P$. By Proposition 2.4, $\bigcap_{m=1}^{\infty}\left(I^{m}+P\right)=P$, i.e., $P$ is closed.

$(2) \Rightarrow(3)$. Since $\{0\}$ is closed, we can assume that $R \subseteq \widehat{R}$. Let $M \in T$. By [2, Theorem 21; p. 421], $M \widehat{R}$ is the closure of $M$ in $\widehat{R}$, hence $M \widehat{R} \cap R$ consists of elements of $R$ which are limits of elements contained in $M$. Since $M$ is closed we get that $M \widehat{R} \cap R=M$.

$(3) \Rightarrow(1)$. Since $M \widehat{R}$ is closed in $\widehat{R}$ and since the map $f: R \rightarrow \widehat{R}$ is continuous, $M$ is closed in $R$ for all $M \in T$. If $I \nsubseteq \cap T$, then $I \nsubseteq M$ for some $M \in T$. But then we have $I^{m}+M=R$ for all integer $m>0$, contradicting the fact that $M$ is closed.

Let $T$ be a finite cover of $R$ and set $J=\cap T$ and $S=R-\cup T$. It is immediate from Lemma 2.1 that the map $A \rightarrow A_{S}$ is injective. Also, the $J$-adic completion of $R$ is the same as the $J R_{S}$-adic completion of $R_{S}$. So we have the following result. 
Theorem 4.2. Any Noetherian ring $R$ can be embedded in a complete semilocal Noetherian ring; moreover, if $R$ is irreducible, then $R$ can be embedded in a complete local Noetherian ring.

If $I$ is an ideal of $R$, we write $\operatorname{dep}(I)$ to stand for $\operatorname{dep}_{I}(R)$.

Theorem 4.3. Let $T=\left\{M_{1}, \ldots, M_{n}\right\}$ be a finite cover of the Noetherian ring $R$ and set $J=\cap T$ and $S=R-\cup T$. Then the $J$-adic completion $\widehat{R}$ of $R$ is a $C$-M ring if and only if $\operatorname{dep}\left(M_{i}\right)=\operatorname{ht}\left(M_{\imath}\right), i=1,2, \ldots, n$.

Proof. To prove the theorem, it suffices to show that ht $\left(M_{i}\right)=\operatorname{ht}\left(M_{i} \widehat{R}\right)$ and $\operatorname{dep}\left(M_{\imath}\right)=\operatorname{dep}\left(M_{\imath} \widehat{R}\right), i=1,2, \ldots, n$.

(1). The proof of ht $\left(M_{\imath}\right)=$ ht $\left(M_{i} \widehat{R}\right)$. Let $B=R_{S}, Q_{i}=M_{\imath} R_{S}$, and $R_{\imath}=B_{Q_{\imath}}$. We now regard $\widehat{R}$ as the $J B$-adic completion of $B$. From [1, Theorem 8.15], $\widehat{R}=\widehat{R}_{1} \times \cdots \times \widehat{R}_{n}$, where $\widehat{R}_{\imath}$ is the completion of the local ring $R_{i}$. By [2, Theorem 30; p. 433] we have

$$
\operatorname{ht}\left(\left(Q_{i} R_{\imath}\right) \widehat{R}_{\imath}\right)=\operatorname{dim}\left(\widehat{R}_{\imath}\right)=\operatorname{ht}\left(Q_{i}\right)=\operatorname{ht}\left(M_{i}\right) .
$$

Thus

$$
\operatorname{ht}\left(M_{i} \widehat{R}\right)=\operatorname{ht}\left(\left(Q_{i} R_{i}\right) \widehat{R}_{i}\right)=\operatorname{ht}\left(M_{i}\right)
$$

(2). The proof of $\operatorname{dep}\left(M_{i}\right)=\operatorname{dep}\left(M_{\imath} \widehat{R}\right)$. We may view $R$ as a subring of $\widehat{R}$. If $A$ is an $R$-module, let $\widehat{A}$ be the $J$-adic completion of $A, z(A)$ and $z(\widehat{A})$ the sets of annihilators of $A$ and $\widehat{A}$ respectively. First we have that if $x \notin z(A)$, then $x \notin z(\widehat{A})$. This is because tensoring $\widehat{R}$ over $R$ preserves the monomorphism $A \stackrel{x}{\longrightarrow} A$, for $\widehat{R}$ is $R$-flat. Let $\operatorname{dep}\left(M_{\imath}\right)=s$ and $x_{1}, \ldots, x_{s}$ be a maximal regular sequence (on $R$ ) contained in $M_{i}$. Since $x_{j+1} \notin z\left(R /\left(x_{1}, \ldots, x_{j}\right)\right)$ implies $x_{j+1} \notin z\left(\widehat{R} /\left(x_{1}, \ldots, x_{j}\right) \widehat{R}\right)$, we have that $x_{1} \ldots, x_{s}$ is a regular sequence on $\widehat{R}$ contained in $M_{i} \widehat{R}$, so $\operatorname{dep}\left(M_{\imath} \widehat{R}\right) \geq s$.

On the other hand, since $M_{\imath} \subseteq z\left(R /\left(x_{1}, \ldots, x_{s}\right)\right)$ and since $M_{i}$ is maximal, there is $x \in R$ such that $M_{i}=\left(x_{1}, \ldots, x_{s}\right):_{R} x$. Thus we have $M_{i} \widehat{R}=$ $\left(x_{1}, \ldots, x_{s}\right) \widehat{R}: \widehat{R} x$, by $\left[\mathbf{2}\right.$, Lemma 7 ; p. 424]. So $M_{i} \widehat{R} \subseteq z\left(\widehat{R} /\left(x_{1}, \ldots, x_{s}\right) \widehat{R}\right)$ and hence $\operatorname{dep}\left(M_{i} \widehat{R}\right)=s=\operatorname{dep}\left(M_{i}\right)$. The proof is complete.

Corollary 4.4. Let $R$ be a semi-local Noetherian ring and $J$ the Jacobson radical of $R$. Then the $J$-adic completion $\widehat{R}$ of $R$ is a $C$-M ring if and only 
if $R$ is a $C-M$ ring.

Corollary 4.5. Any $C$ - $M$ ring can be embedded in a complete semi-local $C-M$ ring.

Acknowledgement. Part of the paper was written while the author was visiting the Department of Mathematics of the University of Stockholm, he would like to thank Ralf Fröberg for his advice and help. He would also like to thank the referee for helpful comments.

\section{References}

[1] H. Matsumura, Commutative ring theory, Cambridge University Press, 1986.

[2] D.G. Northcott, Lessons on rings, modules and multiplicities, Cambridge University Press, 1968.

[3] E. Matlis, Modules with descending chain condition, Trans. Amer. Math. Soc., 97 (1960), 495-508.

[4] A.W. Chatters, C.R. Hajarnavis and N.C. Norton, The Artin radical of a Noetherian ring, J. Austral. Math. Soc., 23 (Series A) (1977), 379-384.

[5] D.E. Rush, Asymptotic primes and integral closure in modules, Quart. J. Math. Oxford (2), 43 (1992), 477-499.

[6] H. Matsumura, Commutative algebra (Second Edition), The Benjamin/Cummings Publishing Company, Inc., 1980.

Received August 9, 1993 and revised January 27, 1994.

HeBei University

BAODING, 071002

People's Republic of China 


\title{
PACIFIC JOURNAL OF MATHEMATICS
}

Founded by

\author{
$\begin{array}{ll}\text { E. F. Beckenbach (1906-1982) F. Wolf (1904-1989) } & \text { F }\end{array}$
}

EDITORS

Sun-Yung A. Chang (Managing Editor) Robert Finn

University of California

Los Angeles, CA 90095-1555

pacific@math.ucla.edu

F. Michael Christ

University of California

Los Angeles, CA 90095-1555

christ@math.ucla.edu

Nicholas Ercolani

University of Arizona

Tucson, AZ 85721

ercolani@math.arizona.edu
Stanford University

Stanford, CA 94305

finn@gauss.stanford.edu

Steven Kerckhoff

Stanford University

Stanford, CA 94305

spk@gauss.stanford.edu

Martin Scharlemann

University of California

Santa Barbara, CA 93106

mgscharl@math.ucsb.edu
Gang Tian

Massachusettes Institute of Technology

Cambridge, MA 02139

tian@math.mit.edu

\section{S. Varadarajan}

University of California

Los Angeles, CA 90095-1555

vsv@math.ucla.edu

Dan Voiculescu

University of California

Berkeley, CA 94720

dvv@math.berkeley.edu

\section{SUPPORTING INSTITUTIONS}

\author{
CALIFORNIA INSTITUTE OF TECHNOLOGY \\ NEW MEXICO STATE UNIVERSITY \\ OREGON STATE UNIVERSITY \\ STANFORD UNIVERSITY \\ UNIVERSITY OF ARIZONA \\ UNIVERSITY OF BRITISH COLUMBIA \\ UNIVERSITY OF CALIFORNIA \\ UNIVERSITY OF HAWAII
}

\author{
UNIVERSITY OF MONTANA \\ UNIVERSITY OF NEVADA, RENO \\ UNIVERSITY OF OREGON \\ UNIVERSITY OF SOUTHERN CALIFORNIA \\ UNIVERSITY OF UTAH \\ UNIVERSITY OF WASHINGTON \\ WASHINGTON STATE UNIVERSITY
}

The supporting Institutions listed above contribute to the cost of publication of this Journal, but they are not owners or publishers and have no responsibility for its contents or policies.

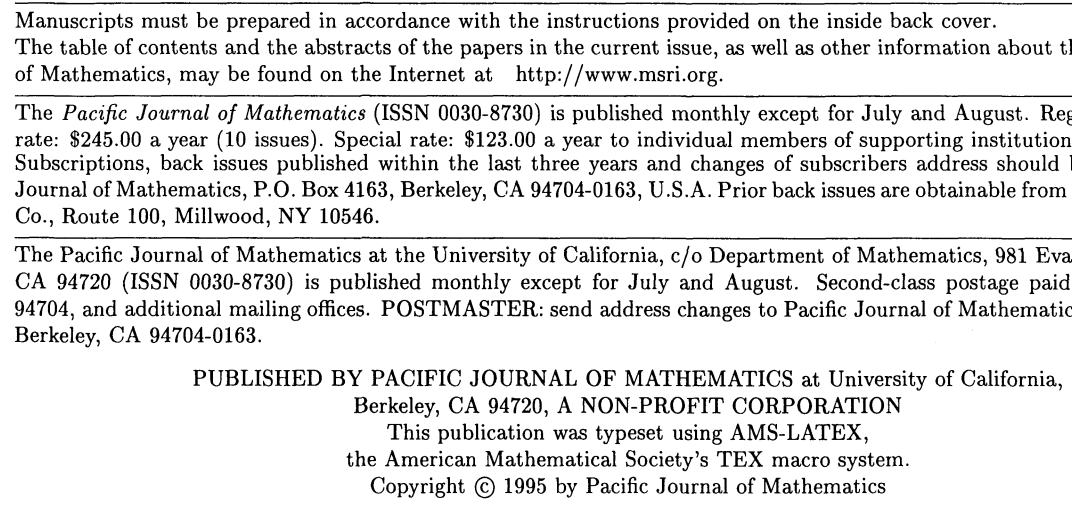




\section{PACIFIC JOURNAL OF MATHEMATICS}

\section{Volume $173 \quad$ No. $1 \quad$ March 1996}

Isometric immersions of $H_{1}^{n}$ into $H_{1}^{n+1}$

KINETSU ABE

Rotationally symmetric hypersurfaces with prescribed mean curvature

MARIE-FRANÇOISE BIDAUT-VÉRON

The covers of a Noetherian module

JIAN-JUN CHUAI

On the odd primary cohomology of higher projective planes

MARK FoSKEY and MiCHAEL DAVID SLACK

Unit indices of some imaginary composite quadratic fields. II

MiKinito HiRABAYASHI

Mixed automorphic vector bundles on Shimura varieties

MIN Ho LeE

Trace ideal criteria for Toeplitz and Hankel operators on the weighted Bergman

spaces with exponential type weights

PENG LIN and RICHARD ROCHBERG

On quadratic reciprocity over function fields

KATHY DONOVAN MERRILL and LYNNE WALLING

$\left(A_{2}\right)$-conditions and Carleson inequalities in Bergman spaces

TAKAHIKO NAKAZI and MASAHIRO YAMADA

A note on a paper of E. Boasso and A. Larotonda: "A spectral theory for solvable Lie 173 algebras of operators"
C. Отт

Tensor products with anisotropic principal series representations of free groups

Carlo Pensavalle and Tim Steger

On Ricci deformation of a Riemannian metric on manifold with boundary

YING SHEN

The Weyl quantization of Poisson $S U(2)$

Albert Jeu-Liang Sheu

Weyl's law for $S L(3, \mathbb{Z}) \backslash S L(3, \mathbb{R}) / S O(3, \mathbb{R})$

ERIC GeORge Stade and Dorothy IRENe Wallace (ANDREOli)

Minimal hyperspheres in two-point homogeneous spaces

PER TOMTER

Subalgebras of little Lipschitz algebras

NiKOLAI ISAAC WEAVER 